\title{
Role of coronary artery calcium score in risk prediction and therapy guidance of asymptomatic individuals
}

\author{
Judit Simon', Lili Száraz', Béla Merkely', Pál Maurovich-Horvatt, ${ }^{1,2}$ \\ ${ }^{1}$ MTA-SE Lendület Cardiovascular Imaging Research Group, Heart and Vascular Center, \\ Semmelweis University, Budapest \\ ${ }^{2}$ Medical Imaging Centre, Semmelweis University, Budapest
}

Address for correspondence:

Judit Simon, MD, e-mail: juditsimon21@gmail.com

Coronary CT angiography (CCTA) has emerged as a gatekeeper to rule out coronary artery disease (CAD), due to its high sensitivity and negative predictive value. Prior to CCTA a native calcium screening scan is acquired, which provides additional information about the coronary artery anatomy and cardiovascular risk prediction by measuring coronary artery calcification (CAC).

Based on large population-based and cohort studies, zero CAC score is linked to low probability of cardiovascular events in the future. Moreover, zero CAC score is superior in the discrimination and risk reclassification when compared with other cardiovascular risk factors. CAC score can also help to identify those who are less likely to benefit from statin pharmacotherapy. Since CAC score has an important role in risk stratification and it is a cheap and widely accessible non-invasive imaging modality, the major guidelines have already incorporated CAC score for risk prediction and therapy guiding. However, these guidelines give slightly different recommendations. Therefore, this review aimed to introduce the CAC measurement and to summarize the prognostic role of CAC scoring in individualized risk prediction and guiding preventative therapies.

Keywords: Coronary CT angiography, coronary artery disease, asympthomatic angina, calcium score, risk prediction

\section{Introduction}

Coronary artery disease (CAD) is a pathological process characterized by atherosclerotic plaque accumulation in the coronary arteries, which may lead to coronary luminal narrowing and ischemia (1). CAD is the most prominent cause of morbidity and mortality in Western societies, accounting for approximately 7.4 million (13\%) deaths per year (2). Since many CAD patients are asymptomatic, the first manifestation is generally myocardial infarction or sudden cardiac death (3). Therefore, there is an emerging need for improved screening tools for subclinical coronary atherosclerosis, which is a strong predictor of future adverse cardiovascular events (4-7). With the help of non-contrast en- hanced low dose cardiac computed tomography (CT) scans, we can quantify the amount of coronary artery calcification (CAC). CAC scoring is a robust method to detect and quantify CAD particularly in those without any symptom (8). This review aimed to summarize the prognostic role of CAC scoring in individualized risk prediction and guiding preventative therapies.

\section{Detection of coronary artery calcification}

Several CAC scoring systems have been described. Among them, the Agatston score is considered as the gold standard for the quantification of CAC. Agatston score is based on the weighted density score given

Received 16. 03. 2020, accepted 25. 08. 2020. 


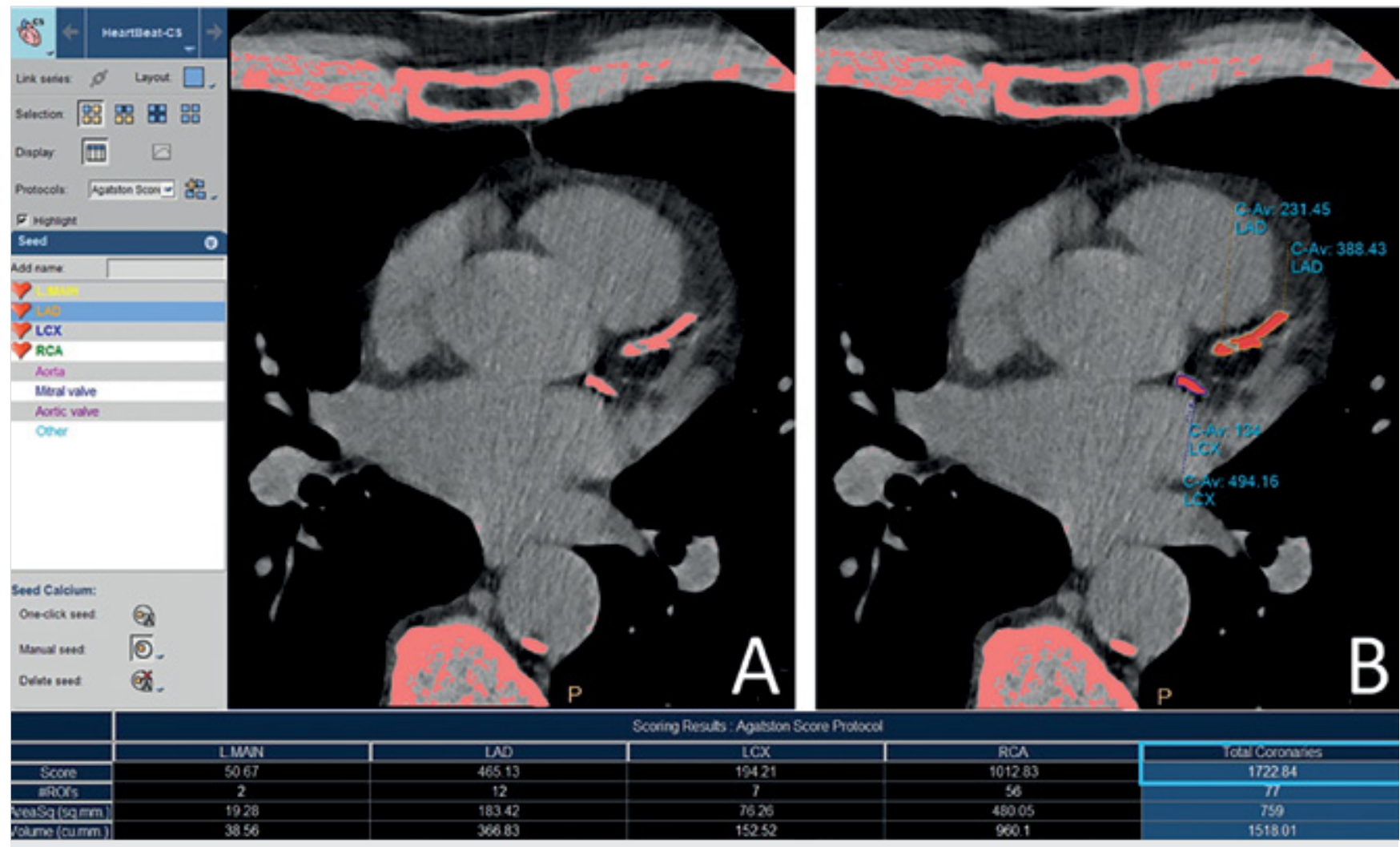

FIGURE 1. CAC score measurement with a semi-automated software. A: As a first step, the software automatically recognize and mark high-density areas of at least $1 \mathrm{~mm}^{2}$-with $>130$ Hounsfied units or $\geq 3$ adjacent pixels (9). B: We can manually assign calcified plaques to the corresponding coronary arteries. The program calculates several vessel-based and total CAC parameters, such as CAC score, area or volume

to the highest attenuation value (HU) multiplied by the area of the calcification speck (9). This scoring system has been adapted to the current multidetector CT, which is still the most widely used imaging modality to detect and calculate CAC score (10). Representative example of a CAC score measurement can be seen in Figure 1. Beyond Agatston score, calcium volume score and relative calcium mass score can be used for the quantification of the extent of coronary artery calcification. Calcium volume score has proven to be the most robust and reproducible method (11). It is calculated by multiplying the number of voxels with calcification by the volume of each voxel, including all voxels with an attenuation $>130 \mathrm{HU}$. However, this method is particularly sensitive to the partial volume effect. Relative calcium mass score is calculated by multiplying the mean attenuation of the calcified plaque by the plaque volume in each image, thus reducing the variation caused by the partial volume. The absolute calcium mass score uses a correction factor based on the attenuation of water (11).

Prognostic value of CAC score

Several population-based and cohort studies have investigated the prognostic value of CAC score in the prediction of CAD.

In 2007 Budoff et al. reported that based on their large cohort study of 25,253 asymptomatic individuals,
CAC was an independent predictor of mortality after adjustment for age, sex, ethnic background, smoking, diabetes mellitus, positive family history for premature CAD, hypertension and hypercholesteraemia. Regarding the 10-year follow-up, in those with zero CAC, the survival was $99.4 \%$, while in those with $>1000$ CAC, it decreased to $87.7 \%(p<0.0001)$ (Figure 2) (12). Another

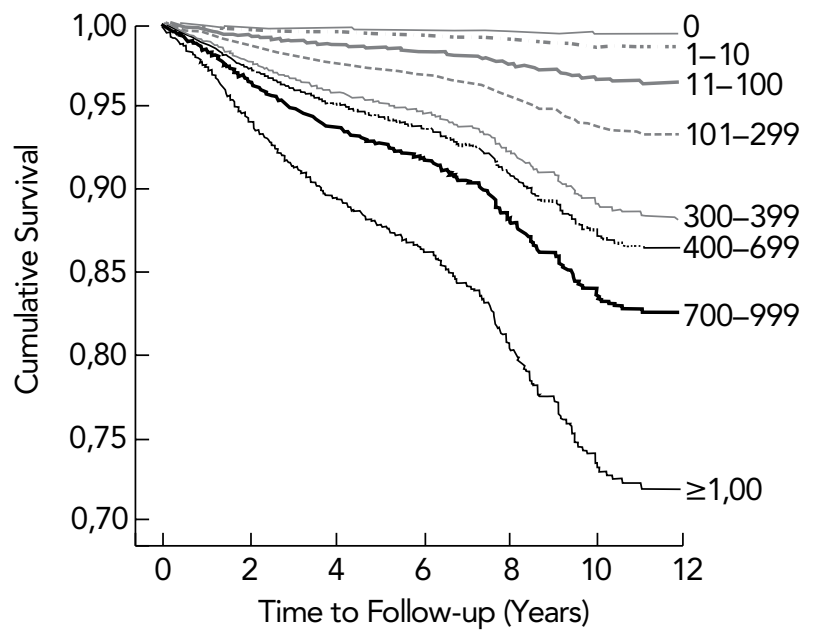

FIGURE 2. Risk-adjusted cumulative survival by CAC score. Adopted from Budoff MJ et al (12) 
cohort study of 9715 asymptomatic patients reported that the extent of CAC could accurately predict 15-year mortality in the study population (13). Based on a large prospective, population-based study of 6809 individuals between 45 and 84 years, the relationship between CAC and CAD was similar in young and elderly participants, suggesting that CAC scoring is a useful tool for risk prediction regardless of age (14).

The prospective cohort MESA (Multi-Ethnic Study of Atherosclerosis) study investigated the distribution of $\mathrm{CAC}$ on the basis of age, sex, ethnic background in individuals without clinically manifest cardiovascular disease. Based on their analysis of 6110 included participants, higher CAC values were measured in men, and CAC score increased with increasing age. Moreover, when studying the sex-, race and age-based differences, whites had the highest percentiles in all age group and Chinese had the lowest percentiles in the oldest age group regardless of sex. In younger participants, Hispanic women and black men had the lowest percentiles (15). This study provided the "CAC Score Reference Values" for the estimation of CAC score based on age, gender and race.

Another MESA Study of 6814 participants aimed to directly compare six risk markers including CAC, carotid intima-media thickness, ankle-brachial index, brachial flow-mediated dilatation, high-sensitivity C-reactive protein and family history of CAD (16). Their results suggest that $\mathrm{CAC}$ is superior in the discrimination and risk reclassification when compared with the other five markers. In 2015 McClelland et al derived and validated a novel risk score for the estimation of 10-years CAD risk (MESA risk score) (17). Beyond traditional Framingham risk factors such as age, sex, HDL cholesterol, total cholesterol, systolic blood pressure, anti-hypertensive medication use, current smoking and diabetes, MESA risk score included CAC score, as well. External validation for testing MESA risk score was conducted in the Heinz Nixdorf Recall Study (HNR) and in the Dallas Heart Study (DHS) populations. Their results have shown that when adding CAC score to the traditional risk factors, C-statistic improved from 0.75 to $0.80(p<0.0001)$. MESA risk score proved to be a very accurate tool for the estimation of 10 -year CAD risk: external validation analysis resulted in

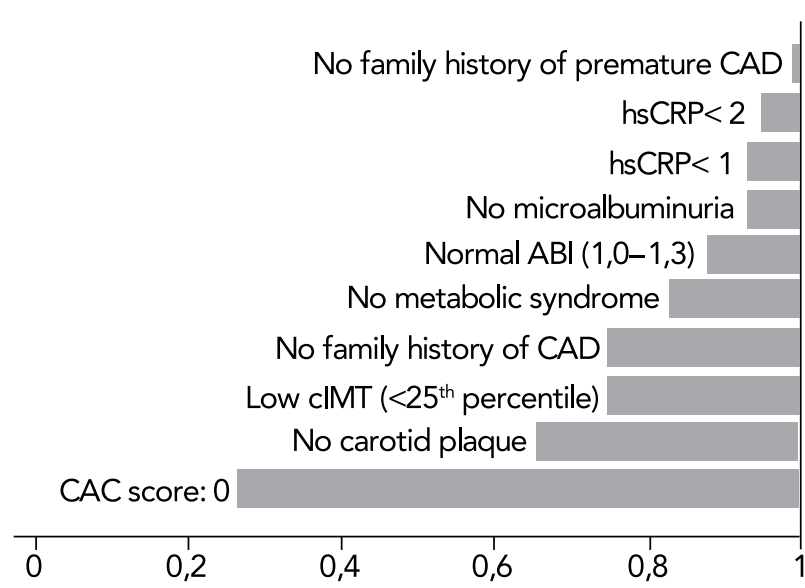

FIGURE 3. Risk factor-adjusted diagnostic likelihood ration of negative risk markers for cardiovascular disease events (26). Abbreviations: ABI: ankle-brachial index; CAC: coronary artery calcium; cIMT: carotid intima-media thickness; hsCRP: high sensitivity C-reactive protein

0.779 Harrell's C-statistic in HNR and 0.816 in DHS. Until now, only MESA risk score incorporated CAC testing in the risk prediction of CAD.

\section{The predictive value of zero CAC score}

Previous studies aimed to investigate the utility of zero CAC score in the risk stratification of patients with suspected CAD (18-25). All of them concluded that zero CAC score is linked to low probability of cardiovascular events in the future.

In a study including 6814 MESA participants, zero CAC score proved to be the strongest among all negative risk markers, with an adjusted mean diagnostic likelihood ratio of $0.14 \pm 0.12$ for CAD (Figure 3). Moreover, in net reclassification improvement analysis, zero CAC score resulted in the largest and most accurate downward risk reclassification (26). Another MESA study of 4758 participants, among those by whom moderate-to-high intensity statin therapy was recommended based on 2013 ACC/AHA guidelines, 41\% had zero CAC and 5.2 atherosclerotic cardiovascular disease event rate of 5.2 per 1000 person-years (23). Absence of CAC was lin-

TABLE 1. Recommendation of the major societies on the use of CAC testing for risk assessment of asymptomatic patients (28-30)

\begin{tabular}{|c|c|c|c|}
\hline & $\begin{array}{l}\text { Class of } \\
\text { recommen- } \\
\text { dation }\end{array}$ & $\begin{array}{l}\text { Level } \\
\text { of evi- } \\
\text { dence }\end{array}$ & Recommendations \\
\hline 2016 ESC & IIb & B & CAC scoring may be considered as a risk modifier for cardiovascular risk assessment. \\
\hline 2017 SCCT & I & B & $\begin{array}{l}\text { It is appropriate to perform CAC scoring for asymptomatic patients without clinical } \\
\text { ASCVD who are between } 40-75 \text { years of age with a ten-year ASCVD risk of } 5-20 \% \text {. }\end{array}$ \\
\hline 2018 ACC/AHA & Ila & B & $\begin{array}{l}\text { CAC testing can be considered between } 40-75 \text { years of age with intermediate risk } \\
\text { to guide statin therapy if risk estimate do not clearly favour initiation of statin therapy. } \\
\text { Preventive pharmacotherapy is recommended }>100 \text { CAC score. }\end{array}$ \\
\hline
\end{tabular}

Abbreviations: ACC: American College of Cardiology; AHA: American Heart Association; ASCVD: atherosclerotic cardiovascular disease; CAC: coronary artery calcium; ESC: European Society of Cardiology; SCCT: Society of Cardiovascular Computed Tomography. 
ked to extremely low CAD rate in the Population-based cohort of JUPITER (Justification for Use of Statins in Prevention: An Intervention Trial Evaluating Rosuvastatin) Study, as well (27).

Therefore, zero CAC score can help to identify those who are less likely to benefit from statin and other preventive pharmacotherapy.

\section{The role of CAC score in primary prevention}

Since CAC screening has emerged as a relatively cheap and widely accessible non-invasive imaging modality, the major guidelines and expert consensus documents have already incorporated CAC score for risk prediction and therapy guiding (Table 1).

The 2016 European Society of Cardiology (ESC) guideline on cardiovascular disease prevention recommends that CAC testing may be considered as a risk modifier for cardiovascular risk assessment (28). The 2017 expert consensus of the Society of Cardiovascular Computed Tomography (SCCT) states that it is appropriate to measure CAC score for shared decision making in patients without symptoms of CAD who are between 40-75 years of age and with $5-20 \%$ ten-year risk of atherosclerotic cardiovascular disease. In those individuals with lower risk, CAC testing may be considered in selected cases, such as in those with family history of premature CAD (29). The 2018 ACC/AHA cholesterol guideline recommends that $\mathrm{CAC}$ scoring can be considered between $40-75$ years of age with intermediate risk to guide statin therapy if risk estimate do not clearly favor initiation of statin therapy. In case of zero CAC, it is reasonable to withhold statin and initiation of preventive pharmacotherapy is recommended for those patients with $>100$ CAC score (30).

The difference in the recommendations of the various societies can be explained by several facts. Since the ESC guideline was published in 2016, it does not include the most recent study results, while the 2018 ACC/AHA cholesterol guideline is based on more recent data supporting the use of CAC in therapy guidance $(18,31-33)$. The expert consensus of the SCCT places the greatest emphasis on the role of CAC score in patient management among the three societies. However, the SCCT document is an expert consensus, which does not have the same requirements as a guideline and therefore it can be more progressive.

In Hungary, unfortunately, CAC scoring is not used for risk stratification in asymptomatic patients despite the strong supporting evidences. Coronary calcium scan is routinely acquired before each CCTA in symptomatic patients with suspected CAD. The non-contrast enhanced scan is used mainly for planning the CCTA image acquisition and in some cases to defer from CCTA due to very high CAC score. High CAC score values are associated not only with elevated cardiovascular risk,

\begin{tabular}{|c|c|c|c|c|c|c|}
\hline \multirow{10}{*}{ 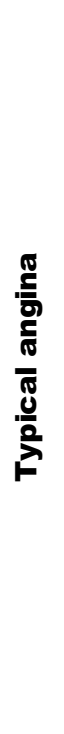 } & \multirow{5}{*}{ 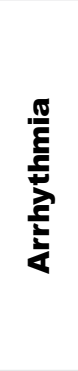 } & 8 & 0 & 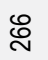 & 唼 & $\stackrel{ఠ}{0}$ \\
\hline & & ○ & 0 & $\bar{్}$ & : & $\bar{g}$ \\
\hline & & R & 吕 & $\stackrel{0}{\stackrel{\rho}{m}}$ & 总 & $\hat{\sim}$ \\
\hline & & 8 & 을 & $\bar{\xi}$ & $\frac{0}{0}$ & $\widetilde{尺}$ \\
\hline & & 요 & $\stackrel{\leftrightarrow}{\circ}$ & 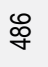 & 莗 & 志 \\
\hline & \multirow{5}{*}{ 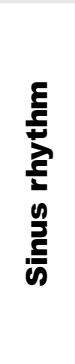 } & ৪ & ஜ & $\stackrel{0}{7}$ & 总 & $\stackrel{0}{R}$ \\
\hline & & ஜ̊ & 욤 & $\bar{f}$ & 品 & స్ల్ \\
\hline & & R & $\stackrel{\text { D̊ }}{\text {. }}$ & ్ָరి & $\stackrel{\frac{\pi}{4}}{2}$ & $\stackrel{\infty}{\infty}$ \\
\hline & & 8 & $\stackrel{\leftrightarrow}{\sim}$ & $\bar{\infty}_{\infty}^{\circ}$ & $\stackrel{8}{\circ}$ & สี \\
\hline & & ㅇํ & $\frac{\circ}{m}$ & $\mathscr{\varnothing}$ & $\underset{\infty}{\mathbb{~}}$ & 鸟 \\
\hline \multirow{10}{*}{$\begin{array}{l}\frac{\pi}{5} \\
\frac{5}{\frac{\pi}{\pi}} \\
\frac{\pi}{\pi} \\
\frac{0}{2} \\
\frac{2}{8}\end{array}$} & \multirow{5}{*}{ 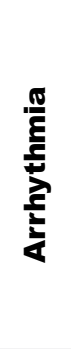 } & \& & $F$ & $\bar{ల}$ & 온 & ฮ్ర \\
\hline & & ஓ & 8 & 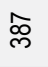 & 莒 & 式 \\
\hline & & ㅇ & $\grave{\Upsilon}$ & $\mathfrak{z}$ & శ్రి & ఠ్ \\
\hline & & 8 & 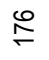 & 字 & ఫ्ञ & $\hat{\infty}$ \\
\hline & & 요 & $\overline{\tilde{N}}$ & 尺్ర్గ & 景 & $\stackrel{m}{\sigma}$ \\
\hline & \multirow{5}{*}{ 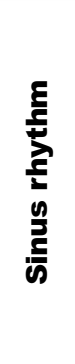 } & \& & 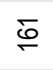 & ঞ̊ & \& & ఖ్ \\
\hline & & : & $\stackrel{\varrho}{\sim}$ & 间 & I & 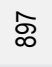 \\
\hline & & R & $\underset{\sim}{\Sigma}$ & శ్ & $\stackrel{\circ}{\triangleright}$ & ్ㅇㅇ \\
\hline & & 8 & ঙ্ల & f & $\check{\infty}^{\infty}$ & $\stackrel{\infty}{\circ}$ \\
\hline & & 웅 & চ্ল & হ & 足 & $\stackrel{\overbrace{}}{\circ}$ \\
\hline \multirow{12}{*}{ 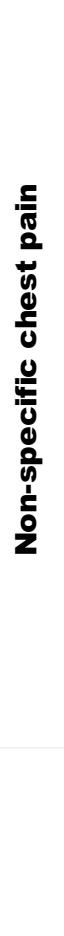 } & \multirow{5}{*}{ 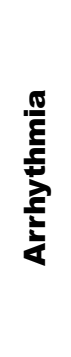 } & ৪ & $\stackrel{8}{ }$ & $\stackrel{œ}{\circ}$ & [? & 郫 \\
\hline & & : & $\because$ & 乎 & ఠ్ & $\bar{\infty}$ \\
\hline & & ㅇ & $\stackrel{\mathscr{R}}{=}$ & 足 & œ & $\stackrel{\circ}{\infty}$ \\
\hline & & 8 & శ్లి & 동 & $\stackrel{\infty}{\sim}$ & $\bar{\sigma}$ \\
\hline & & 움 & $\stackrel{\leftrightarrow}{\sim}$ & 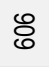 & 劳 & $\hat{\circ}$ \\
\hline & \multirow{5}{*}{ 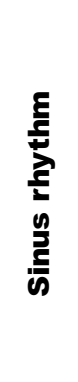 } & ৪ & $\stackrel{20}{\sim}$ & 点 & $\stackrel{\widetilde{N}}{\sim}$ & 迥 \\
\hline & & : & $\stackrel{?}{\sim}$ & Б্ & $\stackrel{\infty}{\gtrless}$ & Бึ \\
\hline & & $R$ & బ్ల & ơ & 芯 & ஜ \\
\hline & & 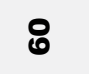 & ఖ్ల & $\overline{2}$ & $\stackrel{\mathscr{ஜ}}{\infty}$ & § \\
\hline & & 오 & $\stackrel{\leftrightarrow}{\%}$ & $\stackrel{\circ}{R}$ & 守 & $\underline{E}$ \\
\hline & & তे & 8 & $\infty$ & 8 & ஜ \\
\hline & & 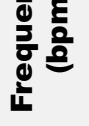 & & & & \\
\hline
\end{tabular}


but they can cause artifacts resulting in non-diagnostic CCTA images. However, there are no clear-cut CAC score thresholds above which performance of CCTA is not recommended therefore it depends on the local expertise and the clinical question to decide if CCTA should be deferred. We have previously determined individualized cut-off values for given probabilities of the need of additional testing, which may facilitate personalized decision-making to perform or defer coronary CTA (Table 2) (34).

CAC screening appears to improve cooperation of asymptomatic patients (21). A meta-analysis of six studies concluded that identification of CAC was associated with significantly increased likelihood of initiating and continuing pharmacologic and lifestyle CAD prevention (35).

\section{Limitations of CAC score-based risk prediction}

Agatston score does not provide information about the total number and regional distribution of calcified plaques, which would be an important aspect of CAD risk prediction (36-38). Moreover, calcified plaques seem to be more resistant to rupture when compared with non-calcified or partially-calcified coronary artery lesions. Therefore, even if increased plaque density contributes to higher CAC score values and increased cardiovascular risk, previous studies have reported that for the same plaque volume, higher calcium density is linked to decreased risk of $\operatorname{CAD}(5,39)$. Similarly, since statins can reduce the volume of non-calcified plaque components, it may lead to higher CAC score parallel with increased plaque stability (ie. paradox calcification) $(40,41)$.

Even if absence of CAC proved to be a strong negative marker, the CAD risk remains high in some special cases. In asymptomatic patients with zero CAC score, after adjustment for age and sex, positive family history for CAD was associated with $73 \%$ increase in CAD (42). Similarly, in those individuals with zero CAC score, mortality hazard ratio was 3.6 (95\% Cl: $2.3-5.7)$ in active smokers compared to non-smokers (43). Moreover, in MACS (Multicenter AIDS Cohort Study), HIV infection was associated with higher prevalence of non-calcified coronary artery plaque independent of other cardiovascular risk factors (44).

\section{Conclusion}

In this review we aimed to summarize the most important data on the prognostic value of CAC scoring in asymptomatic patients and its role in primary prevention. Based on the results of large population-based and cohort studies, CAC scoring is highly encouraged for further risk stratification for decision making on initiation of preventive pharmacotherapy. Moreover, CAC scoring can improve cooperation of patients and adherence to statins. For all these reasons, the major guidelines and expert consensus documents have already incorporated consideration of CAC testing into CAD risk stratification and therapy guiding. However, it is important to keep in mind, that CAC score can only identify calcified plaque components, while non-calcified coronary artery lesions cannot be excluded without CCTA. All in all, given the burden of CAD both on the patients and healthcare, utilizing CAC testing in asymptomatic patients for the purpose of primary prevention and risk stratification should be considered.

\section{Declaration of interest}

The authors have reported that they have no relationships relevant to the contents of this paper to disclose.

\section{References}

1. Knuuti J, et al. 2019 ESC Guidelines for the diagnosis and management of chronic coronary syndromes: The Task Force for the diagnosis and management of chronic coronary syndromes of the European Society of Cardiology (ESC). European Heart Journal 2019; 41(3): 407-477. https://doi.org/10.1093/eurheartj/ehz425

2. Karanasos A LJ WK, Regar E et al. Calcified nodules: an underrated mechanism of coronary thrombosis? JACC Cardiovasc Imaging. 2012;5(10):1071-72 https://doi.org/10.1016/j.jcmg.2012.04.010.

3. Choi EK, et al. Coronary computed tomography angiography as a screening tool for the detection of occult coronary artery disease in asymptomatic individuals. J Am Coll Cardiol 2008; 52(5): 357-65. https://doi.org/10.1016/j.jacc.2008.02.086

4. Chen J, et al. Coronary Artery Calcification and Risk of Cardiovascular Disease and Death Among Patients With Chronic Kidney Disease. JAMA Cardiol 2017; 2(6): 635-643.

https://doi.org/10.1001/jamacardio.2017.0363

5. Criqui $\mathrm{MH}$, et al. Calcium density of coronary artery plaque and risk of incident cardiovascular events. JAMA 2014; 311(3): 271-8. https://doi.org/10.1001/jama.2013.282535

6. Greenland P, et al. Coronary artery calcium score combined with Framingham score for risk prediction in asymptomatic individuals. JAMA 2004; 291(2): 210-5. https://doi.org/10.1001/jama.291.2.210 7. Mitchell JD, et al. Coronary Artery Calcium and Long-Term Risk of Death, Myocardial Infarction, and Stroke: The Walter Reed Cohort Study. JACC Cardiovasc Imaging 2018; 11(12): 1799-1806.

https://doi.org/10.1016/j.jcmg.2017.09.003

8. Paixao AR, et al. Coronary Artery Calcium Improves Risk Classification in Younger Populations. JACC Cardiovasc Imaging 2015; 8(11): 1285-93. https://doi.org/10.1016/j.jcmg.2015.06.015

9. Agatston AS, et al. Quantification of coronary artery calcium using ultrafast computed tomography. J Am Coll Cardiol 1990; 15(4): 827-32. https://doi.org/10.1016/0735-1097(90)90282-t

10. Mao SS, et al. Comparison of coronary artery calcium scores between electron beam computed tomography and 64-multidetector computed tomographic scanner. J Comput Assist Tomogr 2009; 33(2): 175-8. https://doi.org/10.1097/RCT.0b013e31817579ee

11. McCollough $\mathrm{CH}$, et al. Coronary artery calcium: a multi-institutional, multimanufacturer international standard for quantification at cardiac CT. Radiology 2007; 243(2): 527-38.

https://doi.org/10.1148/radiol.2432050808

12. Budoff MJ, et al. Long-term prognosis associated with coronary calcification: observations from a registry of 25,253 patients. J Am Coll Cardiol 2007; 49(18): 1860-70. 10.1016/j.jacc.2006.10.079

13. Shaw LJ, et al. Long-Term Prognosis After Coronary Artery Calcification Testing in Asymptomatic Patients: A Cohort Study. Ann In- 
tern Med 2015; 163(1): 14-21. https://doi.org/10.7326/M14-0612 14. R TM, et al. The relationship of coronary artery calcium to coronary heart disease events is similar in young and elderly participants in the multi-ethnic study of atherosclerosis: a secondary analysis of a prospective population-based cohort. Mayo Clin proc 2014; 89(10): 1350-1359. https://doi.org/10.1016/j.mayocp.2014.05.017

15. McClelland RL, et al. Distribution of coronary artery calcium by race, gender, and age: results from the Multi-Ethnic Study of Atherosclerosis (MESA). Circulation 2006; 113(1): 30-7.

https://doi.org/10.1161/CIRCULATIONAHA.105.580696

16. Yeboah J, et al. Comparison of novel risk markers for improvement in cardiovascular risk assessment in intermediate-risk individuals. JAMA 2012; 308(8): 788-95.

https://doi.org/10.1001/jama.2012.9624

17. McClelland RL, et al. 10-Year Coronary Heart Disease Risk Prediction Using Coronary Artery Calcium and Traditional Risk Factors: Derivation in the MESA (Multi-Ethnic Study of Atherosclerosis) With Validation in the HNR (Heinz Nixdorf Recall) Study and the DHS (Dallas Heart Study). J Am Coll Cardiol 2015; 66(15): 1643-53. https://doi.org/10.1016/j.jacc.2015.08.035

18. Carr JJ, et al. Association of Coronary Artery Calcium in Adults Aged 32 to 46 Years With Incident Coronary Heart Disease and Death. JAMA Cardiol 2017; 2(4): 391-399.

https://doi.org/10.1001/jamacardio.2016.5493

19. Han D, et al. Assessment of Coronary Artery Calcium Scoring for Statin Treatment Strategy according to ACC/AHA Guidelines in Asymptomatic Korean Adults. Yonsei Med J 2017; 58(1): 82-89. https://doi.org/10.3349/ymj.2017.58.1.82

20. Hong JC, et al. Implications of Coronary Artery Calcium Testing for Treatment Decisions Among Statin Candidates According to the ACC/AHA Cholesterol Management Guidelines: A Cost-Effectiveness Analysis. JACC Cardiovasc Imaging 2017; 10(8): 938-952. https://doi.org/10.1016/j.jcmg.2017.04.014

21. Mahabadi AA, et al. CAC Score Improves Coronary and CV Risk Assessment Above Statin Indication by ESC and AHA/ACC Primary Prevention Guidelines. JACC Cardiovasc Imaging 2017; 10(2): 143153. https://doi.org/10.1016/j.jcmg.2016.03.022

22. Mortensen MB, et al. A Simple Disease-Guided Approach to Personalize ACC/AHA-Recommended Statin Allocation in Elderly People: The Biolmage Study. J Am Coll Cardiol 2016; 68(9): 881-91. https://doi.org/10.1016/j.jacc.2016.05.084

23. Nasir K, et al. Implications of Coronary Artery Calcium Testing Among Statin Candidates According to American College of Cardiology/American Heart Association Cholesterol Management Guidelines: MESA (Multi-Ethnic Study of Atherosclerosis). J Am Coll Cardiol 2015; 66(15): 1657-68. https://doi.org/10.1016/j.jacc.2015.07.066 24. Pursnani $A$, et al. Guideline-Based Statin Eligibility, Coronary Artery Calcification, and Cardiovascular Events. JAMA 2015; 314(2): 134-41. https://doi.org/10.1001/jama.2015.7515

25. Yeboah J, et al. Utility of Nontraditional Risk Markers in Individuals Ineligible for Statin Therapy According to the 2013 American College of Cardiology/American Heart Association Cholesterol Guidelines. Circulation 2015; 132(10): 916-22.

https://doi.org/10.1161/CIRCULATIONAHA.115.016846

26. Blaha MJ, et al. Role of Coronary Artery Calcium Score of Zero and Other Negative Risk Markers for Cardiovascular Disease: The Multi-Ethnic Study of Atherosclerosis (MESA). Circulation 2016; 133(9): 849-58.

https://doi.org/10.1161/CIRCULATIONAHA.115.018524

27. Blaha MJ, et al. Associations Between C-reactive Protein, Coronary Artery Calcium, and Cardiovascular Events: Implications for the JUPITER Population From MESA, a Population-Based Cohort Study. Lancet 2011; 378(9792): 684-692. https://doi.org/10.1016/ S0140-6736(11)60784-8

28. Authors/Task Force M, et al. 2016 ESC/EAS Guidelines for the Management of Dyslipidaemias: The Task Force for the Management of Dyslipidaemias of the European Society of Cardiology (ESC) and European Atherosclerosis Society (EAS) Developed with the special contribution of the European Association for Cardiovascu- lar Prevention \& Rehabilitation (EACPR). Atherosclerosis 2016; 253 281-344. https://doi.org/10.1016/j.atherosclerosis.2016.08.018 29. Hecht $\mathrm{H}$, et al. Clinical indications for coronary artery calcium scoring in asymptomatic patients: Expert consensus statement from the Society of Cardiovascular Computed Tomography. J Cardiovasc Comput Tomogr 2017; 11(2): 157-168.

https://doi.org/10.1016/j.jcct.2017.02.010

30. Grundy SM, et al. 2018 AHA/ACC/AACVPR/AAPA/ABC/ACPM/ ADA/AGS/APhA/ASPC/NLA/PCNA Guideline on the Management of Blood Cholesterol: Executive Summary: A Report of the American College of Cardiology/American Heart Association Task Force on Clinical Practice Guidelines. J Am Coll Cardiol 2019; 73(24): 31683209. https://doi.org/10.1016/j.jacc.2018.11.002

31. Budoff MJ, et al. Ten-year association of coronary artery calcium with atherosclerotic cardiovascular disease (ASCVD) events: the multi-ethnic study of atherosclerosis (MESA). Eur Heart J 2018; 39(25): 2401-2408. https://doi.org/10.1093/eurheartj/ehy217

32. Yano Y, et al. Association of Coronary Artery Calcium Score vs Age With Cardiovascular Risk in Older Adults: An Analysis of Pooled Population-Based Studies. JAMA Cardiol 2017; 2(9): 986-994.

https://doi.org/10.1001/jamacardio.2017.2498

33. Malik S, et al. Coronary Artery Calcium Score for Long-term Risk Classification in Individuals With Type 2 Diabetes and Metabolic Syndrome From the Multi-Ethnic Study of Atherosclerosis. JAMA Cardiol 2017; 2(12): 1332-1340.

https://doi.org/10.1001/jamacardio.2017.4191

34. Simon J, et al. Calcium scoring: a personalized probability assessment predicts the need for additional or alternative testing to coronary CT angiography. Eur Radiol 2020. https://doi.org/10.1007/s00330-020-06921-7

35. Gupta A, et al. The Identification of Calcified Coronary Plaque Is Associated With Initiation and Continuation of Pharmacological and Lifestyle Preventive Therapies: A Systematic Review and MetaAnalysis. JACC Cardiovasc Imaging 2017; 10(8): 833-842.

https://doi.org/10.1016/j.jcmg.2017.01.030

36. Arnson $\mathrm{Y}$, et al. Comparison of the Coronary Artery Calcium Score and Number of Calcified Coronary Plaques for Predicting Patient Mortality Risk. Am J Cardiol 2017; 120(12): 2154-2159.

https://doi.org/10.1016/j.amjcard.2017.09.001

37. Blaha MJ, et al. Improving the CAC Score by Addition of Regional Measures of Calcium Distribution: Multi-Ethnic Study of Atherosclerosis. JACC Cardiovasc Imaging 2016; 9(12): 1407-1416. https://doi. org/10.1016/j.jcmg.2016.03.001

38. Tota-Maharaj $R$, et al. Usefulness of regional distribution of coronary artery calcium to improve the prediction of all-cause mortality. Am J Cardiol 2015; 115(9): 1229-34. https://doi.org/10.1016/j.amjcard.2015.01.555 39. Criqui $\mathrm{MH}$, et al. Coronary Artery Calcium Volume and Density: Potential Interactions and Overall Predictive Value: The Multi-Ethnic Study of Atherosclerosis. JACC Cardiovasc Imaging 2017; 10(8): 845-854. https://doi.org/10.1016/j.jcmg.2017.04.018

40. Inoue K, et al. Serial coronary CT angiography-verified changes in plaque characteristics as an end point: evaluation of effect of statin intervention. JACC Cardiovasc Imaging 2010; 3(7): 691-8.

https://doi.org/10.1016/j.jcmg.2010.04.011

41. Motoyama S, et al. Multislice computed tomographic characteristics of coronary lesions in acute coronary syndromes. J Am Coll Cardiol 2007; 50(4): 319-26. https://doi.org/10.1016/j.jacc.2007.03.044 42. Cohen $\mathrm{R}$, et al. Significance of a positive family history for coronary heart disease in patients with a zero coronary artery calcium score (from the Multi-Ethnic Study of Atherosclerosis). Am J Cardiol 2014; 114(8): 1210-4. https://doi.org/10.1016/j.amjcard.2014.07.043 43. McEvoy JW, et al. Mortality rates in smokers and nonsmokers in the presence or absence of coronary artery calcification. JACC Cardiovasc Imaging 2012; 5(10): 1037-45

https://doi.org/10.1016/j.jcmg.2012.02.017

44. Metkus TS, et al. HIV infection is associated with an increased prevalence of coronary noncalcified plaque among participants with a coronary artery calcium score of zero: Multicenter AIDS Cohort Study (MACS). HIV Med 2015; 16(10): 635-9. https://doi.org/10.1111/hiv.12262 